\title{
Les mises en forme de grattoirs carénés / nucléus de l'aurignacien ancien de l'abri Castanet (Sergeac, Dordogne)
}

Carinated scrapers/Early Aurignacian cores shaping of Abri Castanet (Sergeac, Dordogne)

Laurent Chiotti et Catherine Cretin

\section{OpenEdition \\ Journals}

Édition électronique

URL : http://journals.openedition.org/paleo/2077

DOI : $10.4000 /$ paleo.2077

ISSN : $2101-0420$

Éditeur

SAMRA

Édition imprimée

Date de publication : 1 décembre 2011

Pagination : 69-84

ISSN : $1145-3370$

Référence électronique

Laurent Chiotti et Catherine Cretin, «Les mises en forme de grattoirs carénés / nucléus de

l'aurignacien ancien de l'abri Castanet (Sergeac, Dordogne) », PALEO [En ligne], 22 | 2011, mis en ligne

le 14 avril 2012, consulté le 07 juillet 2020. URL : http://journals.openedition.org/paleo/2077 ; DOI : https://doi.org/10.4000/paleo.2077

\section{(c) (†) $\ominus$}

PALEO est mis à disposition selon les termes de la licence Creative Commons Attribution - Pas d'Utilisation Commerciale - Pas de Modification 4.0 International. 


\title{
LES MISES EN FORME DE GRATTOIRS CARÉNÉS / NUCLÉUS DE L'AURIGNACIEN ANCIEN DE L'ABRI CASTANET (Sergeac, Dordogne)
}

\author{
Laurent CHIOTTI(1), Catherine CRETIN ${ }^{(2)}$
}

\begin{abstract}
Résumé : La fouille récente (2005-2010, sous la responsabilité de R. White) des niveaux d'Aurignacien ancien de l'abri Castanet (Sergeac, Dordogne), sur un site ayant déjà connu plusieurs opérations archéologiques successives [fouilles Peyrony, 1911-1913 (Peyrony 1935) ; fouilles Pelegrin, White, 1995-1998 (Pelegrin et White 1998, 1999)], nous confronte aux habituelles questions de représentativité de la zone fouillée, des séries archéologiques exhumées, des comportements observés, etc. Le recours aux collections anciennes (série Peyrony, conservée au Musée national de Préhistoire) permet cependant d'aider à résoudre ponctuellement une question sur une catégorie précise de vestige. C'est le cas des pièces lithiques interprétées comme des mises en forme de grattoirs carénés, toujours trouvées en très faible nombre (ne dépassant jamais la dizaine) dans les séries de fouilles récentes. Ainsi, leur étude sur un effectif supérieur nous permet d'avancer l'existence d'un véritable schéma opératoire de mise en forme d'au moins une partie conséquente des grattoirs carénés de l'Aurignacien ancien de l'abri Castanet.
\end{abstract}

Mots-clés : Aurignacien ancien, Castanet, grattoir caréné, mise en forme, nucléus.

Key-words: Early Aurignacian, Castanet, carinate scrapers, shaping out, core.

\section{Abridged english version \\ Carinated scrapers/Early Aurignacian cores shaping of Abri Castanet (Sergeac, Dordogne)}

The recent excavation (2005-2010, under the supervision of R. White) of the Aurignacian levels at Abri Castanet (Sergeac, Dordogne), in a site that has already undergone several successive archaeological works (excavations by Peyrony, 1911-1913 (Peyrony 1935) ; excavations by Pelegrin, White, 1995-1998 (Pelegrin et White 1998, 1999), confront us with the usual questions of representativeness of the excavated area, the comparison and integration of the excavated archaeological series, old and recent, the behaviours observed from series that are, after all, only samples, etc. To resort to old collections (Peyrony series, stored at the Musée national de Préhistoire and attributed to Aurignacian I and II) nevertheless makes it possible to solve one question about one precise category of artefacts. It is the case of lithic pieces interpreted as "carinate scraper shaping out", always found in small numbers (never more that about ten) in the series from recent excavations. Although already observed at other Aurignacian sites, such as Tuto de Camalhot (Saint-Jean-de-Verges, Ariège), Hui (Beauville, Lot-et-Garonne) or Corbiac-Vignoble II (Bergerac, Dordogne) (Bon 2002) ; Le Brun-Ricalens 2005b ; Bordes 2005), it was indeed difficult to assert that it was a specific shaping out process. The examination of the Peyrony series, with a much larger number of pieces (thirty-nine, plus twenty-five pieces that kept a part of the information about their shaping out, that

(1) Département de Préhistoire du Muséum national d'histoire naturelle, UMR 7194, abri Pataud, 20 rue du Moyen-âge, 24620 Les Eyzies-de-Tayac, France - Ichiotti@mnhn.fr

(2) CNP, MCC / DG1 - UMR 5199 PACEA, Université de Bordeaux, Centre National de Préhistoire, 38 rue du 26 Régiment d'Infanterie, 24000 Périgueux, France - catherine.cretin@culture.gouv.fr 
is sixty-four pieces in all) allows us to confirm the real recurrence of these pieces (although they are not exclusive), to characterize the processes used and their planning. This therefore allows us to propose the existence of a real operational scheme for the shaping out, guided by a very precise volumetric design, of an important part of the carinate scraper (in fact all those that carry still visible shaping out marks) from the Early Aurignacian at Abri Castanet.

The technological observations made on the pieces from the 2005-2011 series as well as the Peyrony series, allow us to detail the following modes of shaping out:

The first step is the choice of the blank of the future carinate scraper/ nucleus that preferentially falls on an object (flake or pebble) showing a sufficient thickness (generally about thirty centimetres) and a smooth and flat surface that will be used as striking platform.

The edges are then shaped with large removals, almost steep, detached with a hard hammer, from the flat surface. Removals aimed at the shaping of the edges are sometime detached from the summit. The shaping may join on the upper part of the piece to form a ridge (triangular section) or surround a residual flat surface (trapezoidal section).

These edges show in more than two-thirds of the cases a second series of flake removals, markedly shorter, steep, called " retouch » because of their strong similarity with the so-called Aurignacian retouch. These removals are subsequent to the large shaping flakes.

To this stage, that is before the start of the débitage, the future flaking surface carries no shaping mark. Following the cases and in function of the morphology of the tip of the piece, the installation of the lamellar flaking surface comes directly or after some preparatory shaping works.

Moreover, even if it is not systematically attested (it seems absent from Abri Pataud, layers 14 to 9, cf. Chiotti 2005), it seems nevertheless that this sequence is a full part of the débitage operational scheme for Early Aurignacian bladelet.

La plupart des « sites classiques » du Paléolithique en Aquitaine ont en commun une longue histoire, non seulement paléolithique, mais aussi archéologique : les interventions successives ont été nombreuses et ont débuté très tôt. Les séries, anciennes ou récentes, triées ou exhaustives, pléthoriques ou limitées, se retrouvent dispersées en de nombreux endroits.

L'abri aurignacien ancien de Castanet, situé dans le Vallon des Roches, lieu-dit Castelmerle, à Sergeac (Dordogne) ne déroge pas à la règle. Dans ce contexte, la problématique d'une réactualisation des données par des fouilles récentes (2005-2010, sous la responsabilité de $R$. White) nous confronte directement à la question de la représentativité de la zone fouillée (terminaison sud de l'abri), de la série récoltée et étudiée et, par conséquent, des comportements techniques observés. II nous reste alors la possibilité de faire appel aux autres séries, notamment la série princeps, afin de compléter nos observations.

L'opération 2005-2010 (White 2007, 2008, 2009 ; White et al. 2010) termine la fouille de l'extrémité sud du gisement, entamée en 1995-1998 (Pelegrin et White 1998, 1999). Dès 1995 , les méthodes de fouille intègrent un tamisage à l'eau systématique. Un seul niveau archéologique, attribué à l'Aurignacien ancien a été mis au jour dans ce secteur.

La série lithique 2005-2010 présente un effectif de 374 outils dont la grande majorité est composée de grattoirs $(33,3 \%)$, grattoirs plats pour la plupart $(24,9 \%)$, souvent sur lame retouchée $(11,1 \%)$. Les grattoirs épais aurignaciens totalisent quant à eux $8,4 \%$ de l'outillage. Les lames retouchées sont nombreuses (27\%, dont 3,6 \% de lames aurignaciennes ou étranglées). Les outils sur lamelle atteignent 10,2\%, soit 38 lamelles retouchées dont de très rares lamelles Dufour. Les burins ne totalisent quant à eux que $1,5 \%$ de l'outillage.

En ce qui concerne les grattoirs carénés, représentés par vingt pièces dont deux doubles et six grattoirs carénés atypiques, il est maintenant largement admis que ces pièces sont en réalité des nucléus à lamelles (voir notamment Rigaud 1993, Lucas 1997, 2000 ; Chiotti 2000, 2003, 2005 ; Bon 2002 ; Le Brun-Ricalens 2005a). Malgré cela, le terme de grattoir caréné reste employé par la majorité des auteurs, et à l'instar de F. Le Brun-Ricalens (2005a), nous pensons qu'il serait prématuré de changer de terminologie, d'autant qu'il a pu être démontré que certaines de ces pièces avaient effectivement été utilisées comme outils (Hays, Lucas 2000). Cependant, afin de souligner le rôle principal de ces objets, nous lui adjoindrons pour la suite de cet article le terme de nucléus, sous la forme : «grattoir caréné / nucléus ».

A l'abri Castanet, l'ensemble des pièces caractéristiques de la chaîne opératoire de production de lamelles sur des grattoirs carénés / nucléus sont présentes (Chiotti et al. 2006). Les lamelles, catégorie de support la plus fréquente dans la série 2005-2010, ont une morphologie indiquant qu'elles sont majoritairement produites sur ce type de nucléus. Le faible nombre de lamelles retouchées ainsi que la présence de lamelles brutes présentant des esquillements nous font penser qu'une grande partie de ces pièces ont été utilisées brutes. (Pelegrin et O'Farrell 2005 ; Chiotti et al. 2006). 
Par ailleurs, cette série présente cinq pièces morphologiquement (gabarit, section) et technologiquement (support, aménagements latéraux) proches des grattoirs carénés / nucléus, sans toutefois présenter d'enlèvements lamellaires. Des objets similaires ont déjà été remarqués ponctuellement et attribués à des " ébauches » ou " préformes $»^{3}$ de grattoirs carénés (voir notamment Bon 2002 ; Bordes et Tixier 2002 ; Bordes 2005 ; Le Brun-Ricalens 2005b). La morphologie de ces pièces les rattache uniquement au morpho-type grattoir caréné, excluant le grattoir à museau. La question de leur interprétation étant soulevée, la série à notre disposition et les publications étant en faible effectif, nous avons tenté d'y apporter une réponse un peu plus argumentée en consultant la série des fouilles Peyrony (1911-1913) conservée au Musée national de Préhistoire des Eyzies.

Nous proposons donc dans cet article de décrire les étapes de mise en forme des grattoirs carénés / nucléus. Nous espérons identifier, le cas échéant, l'existence d'un procédé de mise en forme correspondant à une conception volumétrique précise, intégrée au schéma opératoire de la production lamellaire de l'Aurignacien ancien de l'abri Castanet. La phase de production en ellemême ne sera pas décrite ici.

\section{1 - La question de la mise en forme des grattoirs carénés / nucléus}

Si l'existence d'une mise en forme, antérieure à la production de lamelles a déjà été signalée dans la littérature, elle n'a pas vraiment été décrite, ni considérée comme un procédé particulier, intégré dans la chaîne opératoire du débitage des grattoirs carénés / nucléus.

Lors d'une expérimentation de production de lamelles qu'il réalise avec G. Lucas, seul J. Tixier décrit la mise en forme des grattoirs carénés qu'il fabrique comme " consistant en une sorte de racloir double rectiligne par enlèvements presque abrupts, sans grande application, donnant des négatifs souvent écailleux ou même scalariformes, réservant la partie épaisse de l'éclat pour le débitage de petites lamelles. » (Tixier in Lucas 1999 - p.146).

A vrai dire, les pièces susceptibles d'être rattachées à cette phase sont rares. Elles ont été décrites sur quelques sites du sud de la France. Ainsi, à La Tuto de Camalhot (Saint-Jean-de-Verges, Ariège), F. Bon décrit trois "préformes ", déjà remarquées par le fouilleur J. Vézian au moment de sa découverte comme des «ébauches » de grattoirs carénés : " II s'agit de 2 grands éclats et d'un troisième objet issu d'une fracturation " en split », dont les bords ont été aménagés par des enlèvements détachés à partir de la surface d'éclatement de l'éclat et de la crête établie à l'intersection des bords. Les deux extrémités sont demeurées corticales. 》 (Bon 2002 - p.44).

Le site de Corbiac-Vignoble II (Bergerac, Dordogne ; Tixier 1991) a livré sept nucléus carénés abandonnés au stade de leur mise en forme, qui permettent de confirmer le schéma de préparation du débitage lamellaire décrit par J. Tixier, précédemment cité (Bordes 2005 - p.137). Ce schéma est également confirmé par la présence de remontages et de nucléus abandonnés à différents stades de leur mise en forme (Bordes et Tixier 2002 - p.187). La pièce illustrée dans ces deux publications apparaît comme très semblable à celles que nous avons pu observer dans la série Peyrony de l'abri Castanet (cf. infra).

F. Le Brun-Ricalens signale la présence d'« ébauches » de carénés sur le site de Hui (Beauville, Lot-et-Garonne). II s'agit d'éclats épais, corticaux ou non, ou de nucléus à éclats recyclés, présentant une section triangulaire ou trapézoïdale sur lesquels un dièdre a été aménagé par deux enlèvements adjacents et les bords latéraux ont été régularisés (Le BrunRicalens 2005b - p. 171). L'auteur signale également que certaines de ces "ébauches » évoquent des pièces citées dans la littérature comme des pics ${ }^{4}$.

Les fouilles récentes de l'abri Castanet ont livré des pièces très semblables à celles qui ont été décrites par les auteurs pré-cités. Elles sont présentes dans le matériel des fouilles Pelegrin/White de 1995/1998 (communication personnelle J. Pelegrin) et dans celui des fouilles 2005-2010 (5 pièces, cf. infra).

La conjonction de l'ensemble de ces observations nous a incités à nous interroger sur l'existence d'un procédé de mise en forme d'une partie au moins des grattoirs carénés / nucléus, procédé qui serait suffisamment systématique pour être considéré comme une étape du schéma opératoire de production lamellaire de l'Aurignacien ancien.

\section{2 - Les mises en forme de la série 2005-2010 de l'abri Castanet}

La série issue de la fouille 2005-2010 a livré deux pièces certaines et trois fragmentées présentant tous les éléments caractéristiques de la mise en forme des grattoirs carénés / nucléus.

(3) Les termes d'" ébauche » et de "préforme ", bien que fréquemment employés dans la littérature ne sont pas les mieux adaptés à la réalité technologique des pièces abordées dans cet article. Nous lui préférons l'expression " mise en forme » car elle correspond sans ambigüité à la dernière opération effectuée sur un nucléus avant le débitage proprement dit (Tixier et al. 1980 ; Inizan et al. 1995).

(4) Certaines pièces de l'abri Pataud décrites par l'un d'entre nous comme des pics (Chiotti 1999, 2005) présentent effectivement une morphologie assez comparable aux mises en forme de grattoirs carénés présentées ici. Cependant, à la différence de celles-ci, ces pièces ne présentent généralement pas de surface plane pouvant servir de plan de frappe au grattoir / nucléus ou, lorsqu'elles en présentent une, elles ont des bords très fortement convergents. 
La pièce G13D-143 (fig. 1a) est une mise en forme réalisée sur bloc (plan de frappe néo-cortical). Le support est un ancien éclat roulé de grande dimension en silex sénonien noir $(89$ × 53 × $30 \mathrm{~mm})$. La pièce est aménagée sur ses deux bords par de grands enlèvements qui lui donnent une forme et une section triangulaire. Les aménagements des deux bords ne forment pas de crête, il reste toujours une bande corticale sur le sommet de la pièce. Seuls les bords sont aménagés, l'extrémité destinée à porter la surface de débitage est restée brute (corticale).

La pièce 113C-177 (fig. 1b) est une mise en forme réalisée sur un fragment de bloc plat de silex sénonien noir ( $55 \times 30$ x $28 \mathrm{~mm}$ ), dont l'une des surfaces corticales est utilisée comme plan de frappe. La pièce est taillée dans l'épaisseur d'un galet plat ; un vestige de cortex est visible sur le sommet de la crête supérieure. L'aménagement est réalisé par de grands enlèvements sur les deux côtés de la pièce. Sur un bord (le bord droit sur le dessin ; fig. 1b), il est affiné par l'enlèvement de petits éclats formant une sorte de retouche grossière. La pièce est cassée en son milieu. L'extrémité conservée, encore partiellement corticale, pourrait aussi bien correspondre à l'avant qu'à l'arrière de la pièce.

Cette pièce a fait l'objet d'un remontage relativement complet (21 éléments) ${ }^{5}$ concernant d'une part l'obtention du support à partir du galet et d'autre part la réalisation de la mise en forme.

La pièce 114D-60 (fig. 2a) est cassée. Elle est probablement réalisée sur un éclat, mais les stigmates diagnostiques (talon, bulbe) ont été éliminés par l'aménagement $(63 \times 29 \times 23 \mathrm{~mm})$. Les deux bords de la pièce sont aménagés par le détachement de gros éclats suivi par une " retouche " des bords. La pièce a été cassée probablement au cours de l'aménagement, avant qu'une surface de débitage ait pu être installée.

L'extrémité du fragment retrouvé a été reprise postérieurement par deux enlèvements lamellaires (burin ?).

La pièce G13C-68 (fig. 2b) est une mise en forme possible, réalisée sur un éclat dont la face inférieure a été reprise par des enlèvements très plats $(58 \times 29 \times 24 \mathrm{~mm})$. Le bord droit est aménagé par de grands enlèvements, à partir de la face d'éclatement du support. De petits éclats formant une " retouche " grossière terminent cet aménagement. Le bord gauche est tronqué par un enlèvement longitudinal. Le sommet de la pièce est resté cortical. L'extrémité distale est cassée mais elle présente globalement une morphologie similaire à la pièce $113 \mathrm{C}-177$.

Un éclat d'aménagement du bord gauche, partiellement cortical et antérieur au détachement de l'éclat longitudinal, est remonté sur le sommet de la pièce.
La pièce G13D-63 (fig. 2c) est soit une mise en forme de grattoir caréné / nucléus, soit un grattoir caréné cassé. L'extrémité distale de la pièce, cassée, ne permet pas de savoir si des négatifs d'enlèvements lamellaires étaient ou non présents. Le support est un gros éclat épais (56 x 46 x $25 \mathrm{~mm}$ ) dont les deux bords sont aménagés par de grands enlèvements qui forment une grosse encoche sur chaque bord, en arrière de la cassure.

Quelques « retouches » directes sur l'extrémité gauche de la cassure attestent d'une utilisation de cette pièce après sa fracturation.

Ces deux dernières pièces étant fragmentaires, il n'est pas possible de déterminer si la production lamellaire avait déjà été initiée. En d'autres termes, nous ne pouvons pas dire s'il s'agit de mises en forme cassées ou de grattoirs carénés / nucléus cassés.

Les pièces identifiées dans la série des fouilles 2005-2010 entrent toutes dans le même schéma de préparation. Le support choisi est soit un bloc, plat mais suffisamment épais, présentant une surface très plane utilisée comme plan de frappe pour l'aménagement, soit un gros éclat épais dont la face d'éclatement est utilisée comme plan de frappe. Nous n'avons pas identifié de pièce provenant des premières phases de mise en forme du débitage laminaire parmi les supports utilisés ; les deux types de production semblent donc totalement disjoints.

L'aménagement est généralement similaire sur les deux bords :

1) des détachements de gros éclats sur toute la longueur de la pièce ;

2) des enlèvements plus petits formant une sorte de grosse retouche des bords. Bien que plus grossière, cette « retouche ", abrupte et écailleuse, se rapproche fortement de la retouche aurignacienne.

Le plus souvent, l'aménagement des deux bords se rejoint sur le sommet de la pièce pour former une crête. Cependant, il reste quelquefois une partie corticale ou une surface résiduelle sur le sommet des pièces à la place de cette crête.

Enfin, le fait que deux de ces pièces entrent dans des remontages atteste bien que la production sur grattoir caréné / nucléus est une activité technique réalisée sur place, dans le secteur sud de l'abri Castanet.

\section{3 - Les pièces de la série Peyrony (1911-1913) de l'abri Castanet}

Les fouilles, réalisées par M. Castanet pour D. Peyrony entre 1911 et 1913, ont mis au jour deux niveaux 


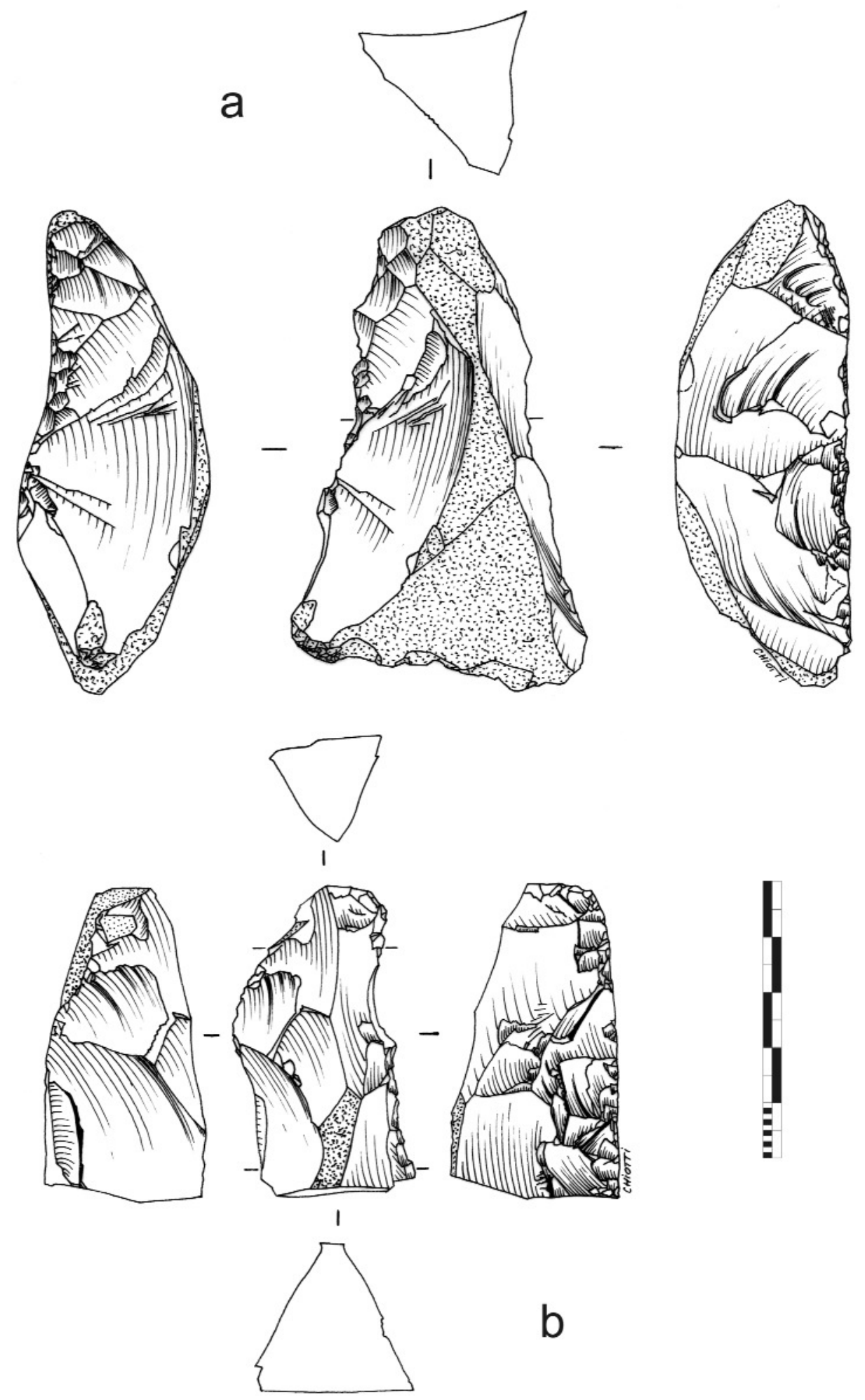

Figure 1 - Mises en forme de grattoirs carénés de l'abri Castanet, fouilles 2005-2010 (dessins L. Chiotti). a : CT/O6 - G13D-143; $b$ : CT/O6 - I13C-177.

Figure 1 - Shaping out of carinate scrapers from abri Castanet, 2005-2010 excavations (drawings by L. Chiotti). a : CT 06 - G13D-143; b : CT 06 - 113C-177. 

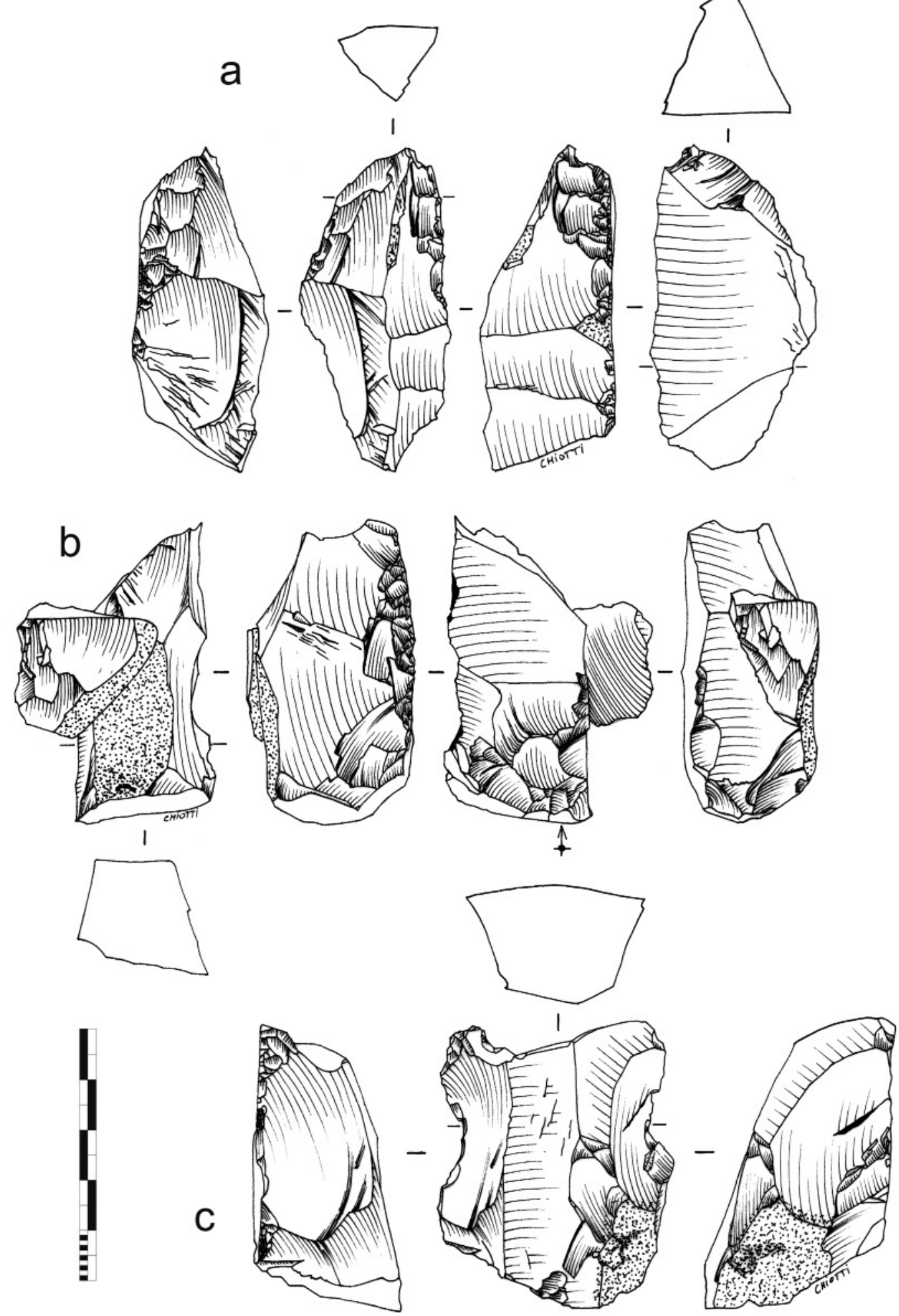

Figure 2 - Pièces fragmentées présentant tous les éléments caractéristiques de la mise en forme des grattoirs carénés / nucléus de l'abri Castanet, fouilles 2005-2010 (dessins L. Chiotti). a : CT/08 - I14D-60 ; b : CT/06 G13C-68 (+ éclat de mise en forme remonté CT/06 - G13D-129) ; c : CT/05 - G13D-63.

Figure 2 - Fragments displaying all of the reduction features characteristic of shaping out from abri Castanet, 20052010 excavations (drawings by L. Chiotti). a: CT/08 - 114D-60; b: CT/06 - G13C-68 (+ refitted reduction flake CT/06 - G13D-129); C: CT/05 - G13D-63. 
archéologiques ( $A$ et $C$ ) séparés par une couche stérile $(B)$. Ces niveaux ont été attribués par $D$. Peyrony à l'Aurignacien I et II, principalement sur la base des fossiles directeurs osseux. La stratigraphie, ainsi qu'une brève description du matériel archéologique seront publiés en 1935 (Peyrony 1935). D. de Sonneville-Bordes en reprendra l'analyse dans sa thèse et dénombrera (tabl. 1), respectivement 1824 outils pour l'Aurignacien I et 1283 pour l'Aurignacien II (Sonneville-Bordes 1960 - p. 244) ${ }^{6}$. Elle mentionnait également l'existence d'une autre série chez M. Castanet.

Conservée au Musée national de Préhistoire, la série Peyrony se présente à l'heure actuelle en trois grands ensembles, respectivement marqués «Aur I » ou « a I 》 (Aurignacien I), «Aur II » ou « a II » (Aurignacien II) et « Aur I/II $\gg^{7}$.

\section{1 - Critères de sélection des pièces étudiées}

Notre choix s'est porté sur l'enregistrement de toutes les pièces qui s'apparentent à des mises en forme dans la série Peyrony, que celles-ci soient attribuées (marquées) à I'Aurignacien I, I/II ou II. Trente-neuf pièces sont ainsi prises en compte, dont vingt-quatre attribuées à l'Aurignacien I, deux à l'Aurignacien I/II et treize à l'Aurignacien II (tabl. 2).

Leur identification s'est faite sur des critères morphologiques et technologiques que nous décrirons par la suite. La présence d'une morphologie propice à l'installation d'une surface de débitage ne peut pas être considérée comme discriminante, ces pièces étant par définition à un stade technique antérieur à sa mise en place.

Les pièces prises en compte lors de la présente étude se situent à divers stades de leur mise en forme. Certaines présentent une mise en forme inachevée. La plupart sont des pièces dont la mise en forme est terminée, mais sur lesquelles le " front de grattoir » n'est pas encore installé. Dans quelques cas, les pièces considérées ne sont plus tout à fait des mises en forme puisqu'on peut déceler sur leur extrémité quelques négatifs d'enlèvements lamellaires, mais sans qu'il y ait un recul notable du débitage. L'intérêt principal de ces pièces, que nous avons qualifiées de mises en forme entamées est de nous fournir la certitude que la mise en forme générale du support est terminée.

Dans le prolongement de cet enregistrement, et afin de collecter les informations disponibles sur des grattoirs carénés qui avaient conservé des traces évidentes de leur aménagement, vingt-cinq grattoirs ont été examinés ${ }^{8}$ (tabl. 2). Parmi ceux-ci se trouvent :

- des grattoirs carénés / nucléus peu exploités, ayant conservé leur morphologie initiale, qui restent (fig. 3b) donc morphologiquement très proches des mises en forme (21 pièces au total, dont 14 Aur I et 7 Aur II) ;

- des grattoirs carénés / nucléus exploités, probablement considérablement raccourcis par rapport à leur morphologie initiale, mais qui ont conservé des traces de leur aménagement (fig. 3a ; 4 pièces Aur l).

En résumé, soixante-quatre pièces issues de la série Peyrony ont été prises en compte dans cette étude, trenteneuf mises en forme et vingt-cinq grattoirs carénés / nucléus sélectionnés parce qu'ils avaient conservé une partie de l'information concernant leur mise en forme. La plus grande partie provient des niveaux attribués à l'Aurignacien I $(n=42)$ et à l'Aurignacien II $(n=20)$, la série marquée Aur $\mathrm{I} / \mathrm{Il}$ étant, quant à elle, représentée uniquement par deux pièces (tabl. 2).

\begin{tabular}{|l|c|c|c|c|c|c|}
\cline { 2 - 7 } \multicolumn{1}{c|}{} & \multicolumn{2}{c|}{$\begin{array}{c}\text { Aurignacien I } \\
\text { (Peyrony) }\end{array}$} & \multicolumn{2}{c|}{$\begin{array}{c}\text { Aurignacien II } \\
\text { (Peyrony) }\end{array}$} & \multicolumn{2}{c|}{ Série 2005-2009 } \\
\cline { 2 - 7 } \multicolumn{1}{c|}{} & Nb. & $\%$ & Nb. & $\%$ & Nb. & $\%$ \\
\hline Grattoirs carénés & 216 & 11,83 & 157 & 12,23 & 18 & 5,64 \\
\hline Grattoirs carénés atypiques & 39 & 2,15 & 17 & 1,32 & 6 & 1,88 \\
\hline Grattoirs à museau & 103 & 5,06 & 105 & 8,19 & 2 & 0,63 \\
\hline
\end{tabular}

Tableau 1 - Comparaison du nombre de grattoirs carénés (et apparentés) entre les séries Peyrony (décomptes D. de Sonneville-Bordes) et la série 2005-2010.

Table 1 - Comparison of numbers of carinate scrapers in the Peyrony assemblage (typological counts by D. de Sonneville-Bordes) and that from 2005-2010.

(6) Lors de son étude, $D$. de Sonneville-Bordes (1960) signalait que les deux séries étaient très proches typologiquement, se rapprochant toutes les deux de la composition d'un Aurignacien ancien. Notre examen rapide des deux séries (l et II) va également dans ce sens.

(7) Cette dernière série, dont nous ne connaissons pas l'origine, ne figure pas dans l'étude de $D$. de Sonneville-Bordes (1960). Selon J. Pelegrin qui l'a étudiée, elle comprend plus de 500 pièces lithiques (communication personnelle J. Pelegrin).

(8) II s'agit ici d'un échantillon de quelques pièces caractéristiques sélectionnées dans un seul but de comparaison. Les données issues de ces pièces n'ont par conséquent aucune valeur quantitative. L'objectif poursuivi dans cette étude étant de préciser les modalités de mise en forme des grattoirs carénés / nucléus avant leur débitage, nous n'y ferons appel que de façon ponctuelle. 


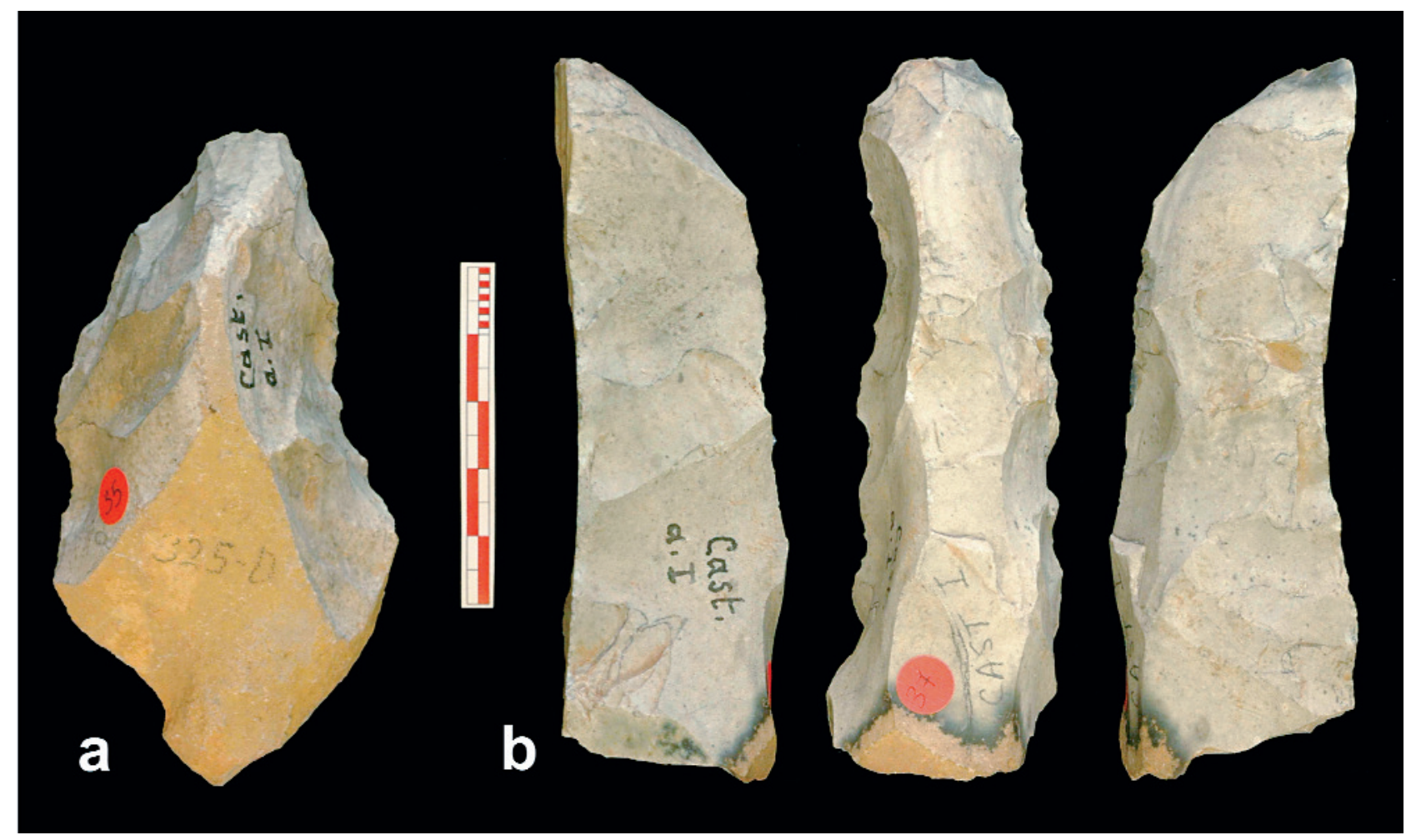

Figure 3 - Grattoirs carénés / nucléus de l'abri Castanet ayant conservé des traces de leur aménagement, fouilles Peyrony (Clichés L. Chiotti). a : grattoir caréné exploité (raccourci), b : grattoir caréné peu exploité (très proche de la mise en forme initiale).

Figure 3 - Carinate scrapers from abri Castanet that have retained traces of their fabrication, Peyrony excavations (photos by L. Chiotti). a: carinate scraper (heavily reduced), b: carinate scraper (slightly reduced and therefore similar to the initial shaping out.

\begin{tabular}{|c|c|c|c|c|c|}
\hline \multirow{2}{*}{\multicolumn{2}{|c|}{ Type de pièce }} & \multicolumn{3}{|c|}{$\begin{array}{l}\text { Attribution culturelle } \\
\text { D. Peyrony }\end{array}$} & \multirow[t]{2}{*}{ TOTAL } \\
\hline & & AUR I & AUR I/II & AUR II & \\
\hline \multirow{4}{*}{$\begin{array}{l}\text { Mise } \\
\text { en forme }\end{array}$} & terminée & 15 & 0 & 5 & 20 \\
\hline & entamée & 7 & 1 & 6 & 14 \\
\hline & partielle & 0 & 0 & 1 & 1 \\
\hline & incertaine & 2 & 1 & 1 & 4 \\
\hline \multicolumn{2}{|c|}{ Total Mises en forme } & 24 & 2 & 13 & 39 \\
\hline \multirow{2}{*}{$\begin{array}{l}\text { Grattoir } \\
\text { caréné }\end{array}$} & $\begin{array}{l}\text { ayant conservé sa morphologie } \\
\text { initiale }\end{array}$ & 14 & 0 & 7 & 21 \\
\hline & $\begin{array}{l}\text { ayant conservé des traces de son } \\
\text { aménagement }\end{array}$ & 4 & 0 & 0 & 4 \\
\hline \multicolumn{2}{|c|}{ Total grattoirs carénés sélectionnés } & 18 & 0 & 7 & 25 \\
\hline \multicolumn{2}{|l|}{ TOTAL } & 42 & 2 & 20 & 64 \\
\hline
\end{tabular}

Tableau 2 - Mises en forme et sélection de grattoirs carénés de la série Peyrony, pris en compte dans cette étude. Table 2 - Shaping out and examples of carinate scrapers from the Peyrony assemblage used in this study. 


\section{2 - Principales caractéristiques des mises en forme}

\section{Matière première et support sélectionné}

Hormis un cas d'indétermination, toutes les pièces sont en silex du Sénonien, majoritairement en silex noir $(n=26$; tabl. 3). Les supports d'origine sont plutôt des éclats $(n=26)$ mais aussi des blocs $(n=13)$, ces derniers pouvant présenter un pan naturel (fracture, diaclase) ou une surface d'éclatement qui sera volontiers exploitée en tant que plan de frappe.

\section{Description morphologique et technologique des mises en forme}

\section{Futur plan de frappe}

La surface destinée à être utilisée comme plan de frappe est dans la très grande majorité des cas $(n=36)$ une surface lisse et unique, constituée par :

- la face inférieure de l'éclat-support $(n=26)$;

- une grande surface d'éclatement qui peut être positive ou négative $(\mathrm{n}=7)$;

- un pan naturel $(n=1)$;

- une surface diaclasique $(n=2)$, antérieurement présente sur les blocs.

Enfin, même si ce cas n'est pas représenté parmi les mises en forme, il existe quelques grattoirs carénés / nucléus pour lesquels cette surface est néocorticale.

Les cas de surfaces constituées de plusieurs négatifs d'enlèvements sont rares $(n=3)$. II s'agit de trois blocs, pour lesquels il n'a sans doute pas été possible de dégager une surface au moyen d'un seul enlèvement.

Ces surfaces $(n=29)$ sont régulières.

\section{Bords}

Les bords des mises en forme sont en grande majorité $(n=33$ ) sub-parallèles (fig. $4 a, c)$, les exceptions étant constituées de bords convergents ( $n=5$; fig. $4 b$ ) et d'une pièce présentant un bord convexe. La délinéation des bords est également assez régulière, les bords sub-parallèles pouvant être parfois sinueux $(n=7)$, ou irréguliers $(n=1)$, mais de façon minoritaire.

Sur les 39 pièces, seuls six bords ne sont pas aménagés, cinq ne présentent qu'un seul bord aménagé, et une ne montre aucune trace d'aménagement sur ses bords. Elle a néanmoins été classée parmi les mises en forme entamées du fait de sa morphologie et de la présence de négatifs d'enlèvements lamellaires. Toutes les autres pièces $(n=33)$ sont aménagées sur leurs deux bords et présentent systématiquement des aménagements à partir de la face inférieure vers le sommet $(n=38)$, lesquels consistent en de grands enlèvements presque abrupts, détachés au percuteur dur et qui couvrent la quasi-totalité de l'épaisseur des pièces.

D'autres enlèvements interviennent parfois dans la mise en forme du grattoir caréné / nucléus. II s'agit d'enlèvements effectués depuis le sommet des pièces $(n=14)$.

Enfin, une deuxième série d'enlèvements directs abrupts, plus petits, plus courts sont très similaires à une retouche écailleuse scalariforme. Ils sont présents dans la majorité des cas, soit sur les deux bords $(n=15)$, soit sur un seul $(n=12)$

\section{Sommet}

Les sections des pièces sont triangulaires $(n=20)$, trapézoïdales $(n=11)$ ou paraboliques/ hémisphériques $(n=4)$. Elles sont parfois mixtes, c'est-à-dire que sur la longueur

\begin{tabular}{|ll|c|c|c|c|}
\hline \multicolumn{2}{|c|}{ Matière première } & $\begin{array}{c}\text { Sénonien } \\
\text { noir }\end{array}$ & $\begin{array}{c}\text { Sénonien } \\
\text { blond }\end{array}$ & $\begin{array}{c}\text { Sénonien } \\
\text { indéterminé } \\
\text { (patiné) }\end{array}$ & $\begin{array}{c}\text { Indéterminée } \\
\text { (forte patine) }\end{array}$ \\
\hline \multirow{3}{*}{$\begin{array}{l}\text { Mise } \\
\text { en forme }\end{array}$} & terminée & 14 & 2 & 3 & 1 \\
\cline { 2 - 6 } & entamée & 11 & 1 & 2 & 0 \\
\cline { 2 - 6 } & partielle & 0 & 0 & 1 & 0 \\
\hline Total & incertaine & 1 & 1 & 2 & 0 \\
\hline
\end{tabular}

\begin{tabular}{|c|c|c|c|c|c|}
\hline \multicolumn{2}{|c|}{ Support } & Eclat & $\begin{array}{c}\text { Eclat } \\
\text { probable }\end{array}$ & Bloc & $\begin{array}{c}\text { Bloc } \\
\text { probable }\end{array}$ \\
\hline \multirow{4}{*}{$\begin{array}{l}\text { Mise } \\
\text { en forme }\end{array}$} & terminée & 11 & 3 & 2 & 4 \\
\hline & entamée & 6 & 1 & 4 & 3 \\
\hline & partielle & 1 & 0 & 0 & 0 \\
\hline & incertaine & 3 & 1 & 0 & 0 \\
\hline \multicolumn{2}{|l|}{ Total } & 21 & 5 & 6 & 7 \\
\hline
\end{tabular}

Tableau 3 - Matières premières et support des mises en forme de grattoirs carénés, série Peyrony.

Table 3 - Raw materials and blank types for carinate scraper shaping out, Peyrony assemblage. 


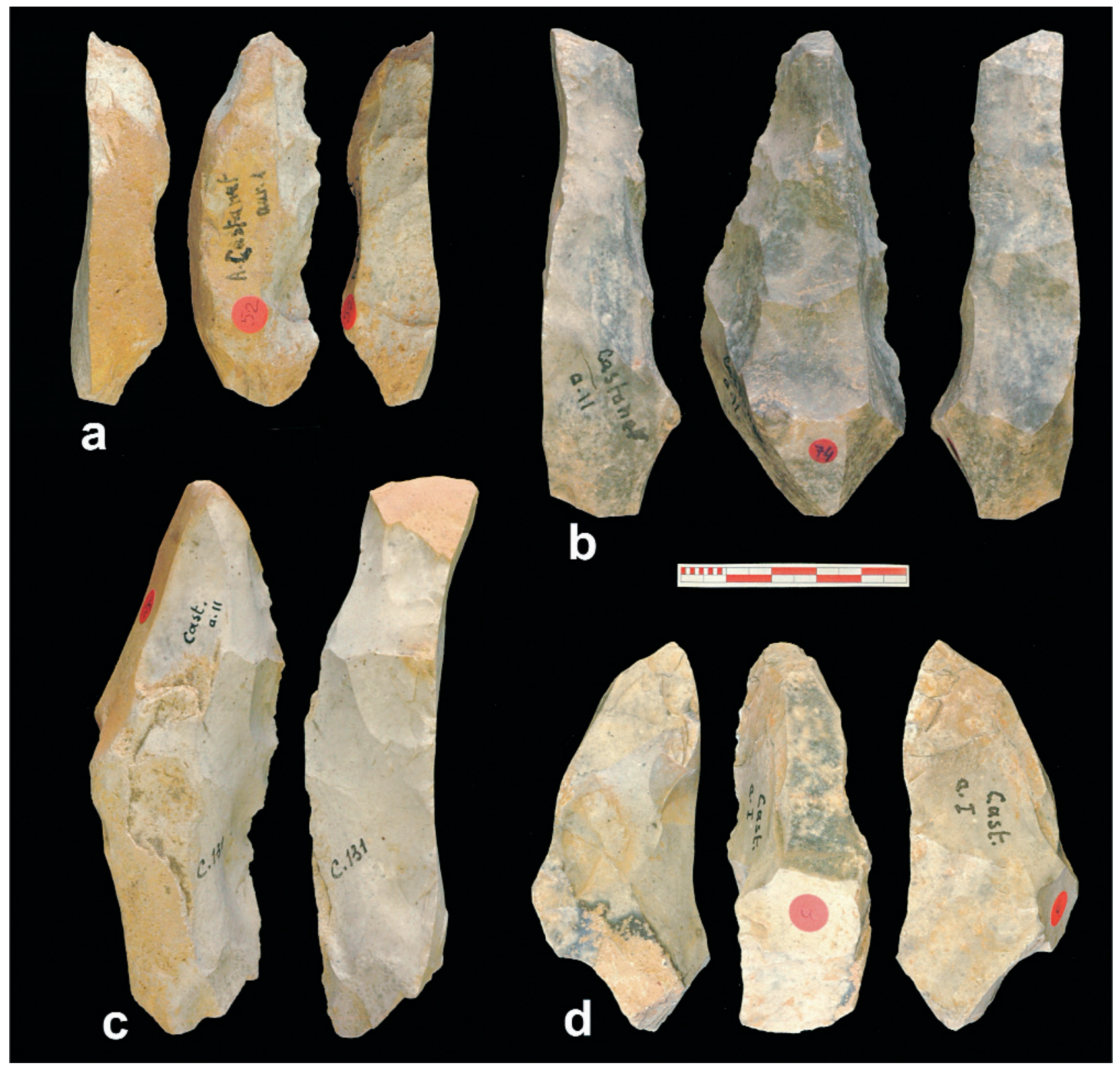

Figure 4 - Mises en forme de grattoirs carénés / nucléus de l'abri Castanet, fouilles Peyrony (Clichés L. Chiotti).

Figure 4 - Shaping out of carinate scrapers from abri Castanet, Peyrony excavations (photos by L. Chiotti).

de la pièce, deux sections peuvent se succéder $(n=4)$. Leurs parties supérieures, qualifiées de sommet, sont donc soit naturelles (arêtes, dômes, faces planes ; $n=19$ ), soit aménagées (crêtes et enlèvements plats transversaux ; $n=20$ ).

\section{Orientation}

Pour les vraies mises en forme, il est parfois possible d'orienter les pièces, c'est-à-dire de savoir de quel côté se situera le débitage de lamelles. Dans d'autres cas, l'orientation n'est pas possible : sur certaines pièces à bord sub-parallèle, le débitage pourrait en effet aussi bien être effectué sur l'une ou l'autre des extrémités, voire sur les deux (grattoir double).

Dans le cas des quatorze mises en forme entamées, les pièces peuvent être orientées, à l'exception de deux d'entre elles qui semblent, dès l'origine, être doubles. Sept d'entre elles présentent au moins un enlèvement lamellaire. 


\section{Modalités de réalisation de la mise en forme}

D'après les observations effectuées, la lecture technologique de ces pièces nous permet de préciser les modalités de mise en forme (fig. 5) :

1 - Une première étape, cruciale, réside dans le choix du support du futur grattoir caréné / nucléus : il se porte de préférence sur un objet présentant une surface lisse et plane qui sera utilisée comme plan de frappe (= face inférieure du grattoir). II peut s'agir de la face inférieure d'un éclat, d'une grande surface d'éclatement ou d'un pan naturel (face corticale plane, diaclase...).

Cette pièce doit également présenter une épaisseur suffisante pour installer à ses dépens une surface de débitage lamellaire : généralement autour d'une trentaine de millimètres.

2 - Les bords sont ensuite aménagés au moyen de grands enlèvements presque abrupts, détachés à partir de cette grande surface utilisée comme plan de frappe, par percussion directe au percuteur dur. Les rares bords qui ne sont pas aménagés présentent des pans réguliers et une inclinaison similaire à celle des grands enlèvements (comprise entre 60 et 85 degrés).

Les aménagements des deux bords peuvent se rejoindre en partie supérieure de la pièce pour former une crête, ce qui lui donne une section triangulaire (fig. $5: 2 b$ ). Dans d'autres cas, ils encadrent une surface supérieure plane résiduelle (fig. $5: 2 a$ ). Cette dernière peut être une surface préexistante (plage corticale, surface diaclasique ou négatif d'enlèvements antérieurs). Elle peut aussi être aménagée par un ou plusieurs enlèvements transversaux à partir d'un bord (cf. infra). La pièce présente alors une section trapézoïdale (fig. $5: 2 \mathrm{c}$ ).

3 - Des enlèvements destinés à l'aménagement des bords sont parfois détachés à partir du sommet par percussion directe au percuteur dur (fig. 5 : 3a). Ceux-ci sont le plus souvent postérieurs aux enlèvements issus de la face inférieure, cela ayant été déterminé dans dix cas sur quatorze. II n'a pas été possible, dans les autres cas, de déterminer l'ordre des enlèvements provenant des faces inférieures et supérieures de la pièce, à l'exception d'une mise en forme entamée présentant clairement une intercalation entre les enlèvements issus de la face inférieure et du sommet.

Nous n'avons trouvé aucun exemple dont la mise en forme depuis la face supérieure soit antérieure à celle des bords. Cette intervention depuis le sommet s'avère même risquée, puisque l'une des pièces semble avoir été abandonnée à cause d'un enlèvement outrepassé ayant enlevé une grande partie du bord droit. Cela semble également être le cas pour au moins une des pièces de Corbiac-Vignoble II (Bordes 2005 - fig. 9).

Des aménagements transversaux peuvent aussi être présents sur le sommet de la pièce, c'est-à-dire depuis la partie supérieure de l'un des bords (= partie distale d'un négatif d'enlèvement) de la pièce en direction de l'autre bord (fig. $5: 3 b)$. Tous les cas déterminés $(n=5)$ s'avèrent eux aussi postérieurs aux enlèvements depuis la face inférieure. Des négatifs d'enlèvements transversaux antérieurs sont parfois observés (4 cas), mais ils semblent nettement antérieurs et plus liés à l'histoire du support avant sa mise en forme.

Nous inclinons à penser que les deux types d'aménagement procèdent de la même logique : une fois que la régularité, le sub-parallélisme et l'angulation abrupte des bords sont aménagés par les grands enlèvements depuis la face inférieure, l'attention est portée sur le sommet de la pièce, dans l'optique de favoriser un recul aussi régulier que possible du futur " front de grattoir ». En fonction de la morphologie initiale du bloc et de sa section (triangulaire ou quadrangulaire), l'option est choisie d'intervenir depuis le sommet ou bien depuis la partie distale d'un négatif d'enlèvement latéral.

4 - Les bords des mises en forme présentent également dans plus de deux tiers des cas $(n=27)$ une deuxième série d'enlèvements écailleux, nettement plus courts mais tout aussi abrupts, très similaires à ce que nous avons coutume de qualifier de « retouche ». Cette dénomination s'applique d'autant mieux que, quand elle existe, la délinéation de la « retouche » affecte la totalité du bord concerné (une seule exception). Comme cela avait déjà été observé sur les pièces de la série 2005-2010, cette " retouche » s'apparente à la retouche dite aurignacienne

Ces enlèvements sont postérieurs aux grands aménagements effectués depuis les bords. Ils sont en général également postérieurs aux aménagements depuis la face supérieure (seul un cas de recouvrement direct a pu être observé).

Cette « retouche » est destinée à régulariser la délinéation des bords, lesquels restent tout de même relativement sinueux. Elle permet aussi d'obtenir un angle sensiblement plus abrupt.

Douze mises en forme ne comportent pas de traces de cette "retouche ", six d'entre elles sont entamées. Cette dernière remarque implique que la "retouche ", quoique très présente, n'est pas systématique avant l'entame du débitage.

En revanche, sa présence démontre qu'elle peut être en place dès la mise en forme et qu'elle ne résulte pas forcément, comme nous aurions pu le penser, d'un entretien au cours du débitage.

A ce stade, c'est-à-dire avant l'entame du débitage, la future surface de débitage ne porte aucune trace d'aménagement.

5 - Cette étape correspond à l'installation de la surface de débitage lamellaire. Suivant les cas et en fonction de la morphologie de l'extrémité de la pièce, celle-ci peut être installée directement ou bien après quelques aménagements préalables. II existe en effet 8 mises en forme dont aucune extrémité ne semble convenir à l'installation d'une surface de débitage lamellaire. Leur entame aurait donc nécessité un tel aménagement. 


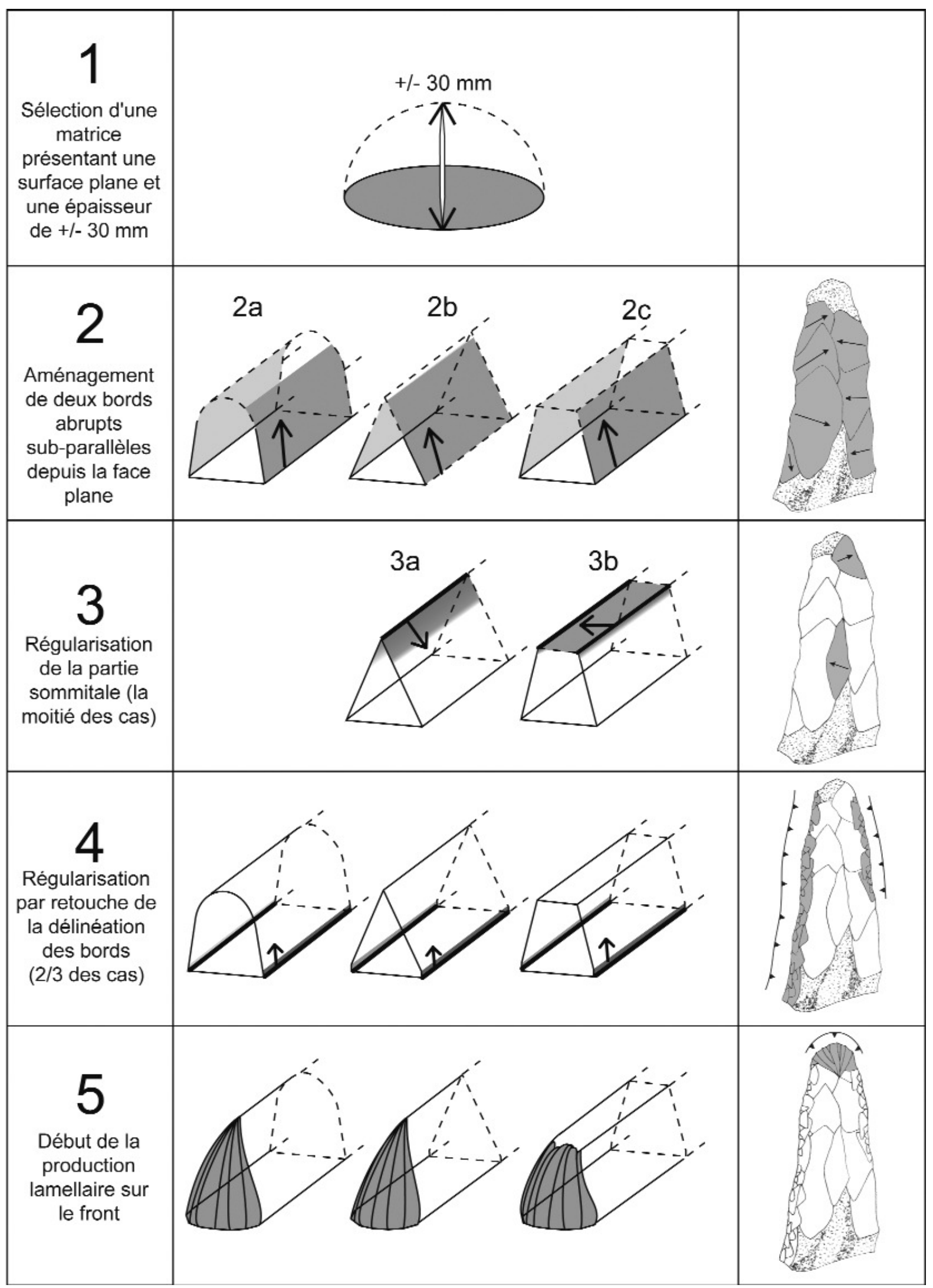

Figure 5 - Modalités de réalisation de la mise en forme.

Figure 5 - Modalities of the shaping of carinate scrapers. 


\section{4 - Synthèse et Conclusions}

Les pièces de la série 2005-2010 peuvent donc être retenues, dans le schéma que nous avons défini à partir de l'étude des pièces de la série Peyrony, comme des mises en forme de grattoirs carénés / nucléus.

Les modalités de mise en forme utilisées à l'abri Castanet aboutissant à la création de pièces dont la morphologie est normalisée, elles révèlent l'existence d'une conception volumétrique très précise, comparable à la " structure volumétrique pérenne » observée par F. Bon (2002 - p. 44). Cette gestion du volume est préalable à l'installation de la surface de débitage lamellaire. Sa réalisation se fait principalement aux dépens des deux bords, à partir de la surface inférieure. Des enlèvements en partie supérieure interviennent parfois dans un second temps (3a et $3 \mathrm{~b})$ afin d'en régulariser la morphologie. Les autres surfaces (extrémités, surface inférieure) ne sont pas affectées par la mise en forme.

Ce système de construction et de gestion préalable du volume autorise, par la suite, l'installation de la surface de débitage lamellaire sur une extrémité, au moyen d'un aménagement limité, parfois même inexistant. Le recul de la surface de débitage nécessite alors un entretien minimum qui se limite à :

- la surface de débitage elle-même ;

- l'intersection surface de débitage / bords pour le maintien du cintrage (éclats et éclats lamellaires latéraux) ;

- l'intersection surface de débitage / sommet pour le maintien de la carène (grandes lamelles) ;

- le contrôle de l'arête sommitale.

Cet entretien est dès lors totalement intégré dans le débitage lamellaire.

Grâce au croisement des données issues des différentes séries de l'abri Castanet, nous avons donc défini un morpho-type de mises en forme de grattoir caréné / nucléus. Tous les grattoirs carénés du site ne peuvent pas être rattachés à ce schéma opératoire de mise en forme : certains n'en présentent aucune, d'autres étant trop réduits pour que les premières phases d'aménagement soient identifiables. A cela s'ajoute la question du recouvrement de l'aménagement par des éclats de ravivage.

En revanche, quelles que soient les séries de l'abri Castanet que nous avons examinées (série Peyrony 19111913 et série White 2005-2010), lorsque des traces de mise en forme sont encore identifiables, elles se rattachent toujours à ce schéma opératoire.

Il reste à préciser la valeur du procédé de mise en forme que nous venons de décrire : est-ce propre à l'abri Castanet ? En d'autres mots une séquence de mise en forme similaire peut-elle être observée sur d'autres sites.

On retrouve ce morpho-type dans d'autres sites, comme par exemple la Tuto de Camalhot (Saint-Jean-de-Verges, Ariège), Hui (Beauville, Lot-et-Garonne) ou Corbiac-
Vignoble II (Bergerac, Dordogne) (Bon 2002 ; Le BrunRicalens 2005b ; Bordes 2005). Malgré ce nombre restreint de points de comparaison, on peut déjà remarquer qu'il existe des mises en forme de grattoirs carénés sur support laminaire à Corbiac-Vignoble II (Bordes et Tixier 2002 ; Bordes 2005 - p.137) et sur des nucléus à éclats recyclés à Hui (Le Brun-Ricalens 2005b - p.171). Cependant, dans les deux cas, si le choix du support diffère, la mise en forme semble similaire.

Ces quelques éléments nous amènent à penser que cette séquence technique fait pleinement partie du schéma opératoire de débitage de lamelles de l'Aurignacien ancien. II ne s'agit pas d'un comportement anecdotique, ni d'une simple convergence technique ou morphologique.

En revanche, en l'état actuel de nos connaissances, il est possible de dire que certaines séries d'Aurignacien ancien ne présentent pas ce procédé de mise en forme. C'est le cas des couches 14 à 9 de l'abri Pataud, où aucune pièce correspondante n'a pu être identifiée (Chiotti 2005).

II semble donc que la séquence de mise en forme décrite ci-dessus ne corresponde pas à un contexte économique particulier d'approvisionnement en matériaux lithiques. En effet, il est présent à l'abri Castanet, et absent à l'abri Pataud, alors que ces deux sites sont dans un environnement similaire. Au contraire, il est attesté dans des contextes économiques différents : atelier de taille à Corbiac Vignoble II et habitat en vallée de la Vézère à l'abri Castanet. Enfin, il est délicat de comparer les finalités économiques des productions lamellaires, principalement à cause de la dimension des pièces et de leur trop fort assujettissement aux conditions de fouille.

Pour finir, ajoutons qu'il nous faut reconnaître que nous ne disposons pour l'instant d'aucune donnée fonctionnelle sur les grattoirs carénés / nucléus de l'abri Castanet : nous n'avons pas relevé de macrotraces d'utilisation et les premières observations tracéologiques de M. Hays sur le matériel archéologique de la série 2005-2010 (étude en cours) se sont avérées infructueuses.

Elargir la comparaison à d'autres séries de l'Aurignacien ancien pourra s'avérer difficile dans bien des cas, dans la mesure où ce schéma de mise en forme, maintenant mieux caractérisé, ne peut être identifié que sur les grattoirs carénés qui ne sont pas trop réduits par le débitage des lamelles. Dans le cas contraire, l'identification devient difficile, voire impossible.

L'extension de la comparaison à d'autres types de pièces carénées et d'autres périodes paléolithiques est tentante, mais en l'état actuel de nos connaissances, elle exige la plus grande prudence car, pour l'instant, elle ne se base que sur une convergence morphologique (la présence d'une carène), voire technologique (l'utilisation d'une petite surface triangulaire doublement convexe pour installer une surface de débitage lamellaire). La présence d'un véritable schéma opératoire au service d'une construction volumétrique récurrente reste à démontrer pour la plupart d'entre elles. 
Le développement et la poursuite des analyses technologiques de séries de l'Aurignacien ancien permettront peut-être de déterminer s'il existe d'autres types de mise en forme des grattoirs carénés. Pour l'heure, en approfondissant la description des procédés techniques mis en œuvre, nous espérons avoir contribué à une meilleure compréhension de l'ensemble du système technique aurignacien, en mettant en évidence l'importance de la production lamellaire dans le secteur sud de l'abri Castanet. Cette importance est certes numérique, mais aussi techno-économique puisqu'elle implique une phase de mise en forme comprenant plusieurs étapes, voire même conceptuelle, puisqu'elle sert à la réalisation et la conservation tout au long du débitage d'une même structure volumétrique : celle d'un objet allongé, épais, de section triangulaire ou subtriangulaire, suffisamment régulier pour permettre un recul du débitage sans changement morphologique notoire.

\section{Remerciements}

Nous tenons à remercier Randall White pour la confiance dont il a fait preuve en nous confiant l'étude du matériel lithique de l'abri Castanet, Jean-Jacques Cleyet-Merle pour nous avoir permis l'accès à la série Peyrony (avec l'accueil toujours chaleureux de André Morala, Peggy Jacquement et Bernard Nicolas) et Jacques Pelegrin pour les informations qu'il a bien voulu nous communiquer sur son travail. Nous remercions également Amy Elizabeth Clark et Stéphane Hoerlé ainsi que les relecteurs, dont Jacques Tixier. L'opération archéologique de l'abri Castanet ne pourrait avoir lieu sans le financement de la Direction Régionale des Affaires Culturelles d'Aquitaine et de la National Science Foundation, USA.

\section{Références bibliographiques}

BON F. 2002 - L'Aurignacien entre Mer et Océan. Réflexion sur l'unité des phases anciennes de l'Aurignacien dans le sud de la France. Paris : Société préhistorique française, 253 p. (Mémoire de la SPF, XXIX)

BORDES J.-G. 2005 - La séquence aurignacienne du Nord de l'Aquitaine : variabilité des productions lamellaires à Caminade-Est, Roc-de-Combe, Le Piage et CorbiacVignoble II. In : F. Le Brun-Ricalens, J-G. Bordes, F. Bon (ed.), Productions lamellaires attribuées à l'Aurignacien : chaînes opératoires et perspectives techno-culturelles. XIV'ime congrès de I'Union internationale des sciences préhistoriques et protohistoriques, Liège (2-8 septembre 2001). Luxembourg : MNHA, p. 123-154 (ArchéoLogiques, 1).

BORDES J.-G., TIXIER J. 2002 - Sur l'unité de l'Aurignacien ancien dans le Sud-Ouest de la France : la production des lames et des lamelles. In : F. Bon, J.-M. Maillo Fernandez, D.Ortega i Cobos (ed.), Autour des concepts de Protoaurignacien, d'Aurignacien archaïque, initial et ancien. Unité et variabilité des comportements techniques des premiers groupes d'hommes modernes dans le Sud de la France et le Nord de l'Espagne. Tableronde de Toulouse (27 février - $1^{\text {er }}$ mars 2003). Madrid :
UNED, p. 175-194 (Espacio, Tiempo y Forma, Serie I, Prehistoria y Arqueologia, t. 15).

CHIOTTI L. 1999 - Remontage d'un pic dans l'Aurignacien ancien de l'abri Pataud (Les Eyzies-de-Tayac, Dordogne). Préhistoire du sud-ouest, 6 (1), p 57-77.

CHIOTTI L. 2000 - Lamelles Dufour et grattoirs aurignaciens (carénés et à museau) de la couche 8 de l'abri Pataud, Les Eyzies-de-Tayac, Dordogne. L'Anthropologie, 104 (2), p. 239-263.

CHIOTTI L. 2003 - Les productions lamellaires dans l'Aurignacien de l'Abri Pataud, Les Eyzies-de-Tayac (Dordogne). Gallia Préhistoire, 45, p. 113-156.

CHIOTTI L. 2005 - Les industries lithiques aurignaciennes de l'abri Pataud, Dordogne, France. Les fouilles de Hallam L. Movius Jr. Oxford : Archeopress, 349 p. (BAR International Series, 1392)

CHIOTTI L., CRETIN C., MORALA A. 2006 - Etude du matériel lithique du secteur sud de l'abri Castanet. Campagnes 2005-2006. In : R. White (dir.), Abri Castanet, Secteur Sud (Commune de Sergeac, Dordogne), rapport de fouille programmée, année 2006 (première année de l'autorisation triennale 2006-2008). Rapport remis au SRA Aquitaine, p. 91-114.

HAYS M. A., LUCAS G. 2000 - A Technological and Functional Analysis of Carinates from Le Flageolet I, Dordogne, France. Journal of Field Archaeology, 27 (4), p. $455-465$.

INIZAN M.-L., REDURON M., ROCHE H., TIXIER J. 1995 Technologie de la pierre taillée. Meudon : Cercle de recherches et d'études préhistoriques, 199 p. (Préhistoire de la Pierre taillée, 4).

LE BRUN-RICALENS F. 2005a - Chronique d'une reconnaissance attendue. Outils "carénés ", outils « nucléiformes »: nucléus à lamelles. Bilan après un siècle de recherches. In : F. Le Brun-Ricalens, J-G. Bordes, F. Bon (ed.), Productions lamellaires attribuées à l'Aurignacien : chaînes opératoires et perspectives technoculturelles. XIV'̀me congrès de I'Union internationale des sciences préhistoriques et protohistoriques, Liège (28 septembre 2001). Luxembourg : MNHA, p. 23-72 (ArchéoLogiques, 1).

LE BRUN-RICALENS F. 2005b - Reconnaissance d'un « concept techno-culturel » de l'Aurignacien ancien ? Modalités, unités et variabilités des productions lamellaires du site d'Hui (Beauville, Lot-et-Garonne, France) : significations et implications. In : F. Le Brun-Ricalens, J-G. Bordes, F. Bon (ed.), Productions lamellaires attribuées à l'Aurignacien : chaînes opératoires et perspectives technoculturelles. XIV'̀me congrès de l'Union internationale des sciences préhistoriques et protohistoriques, Liège (28 septembre 2001). Luxembourg : MNHA, p. 157-190 (ArchéoLogiques, 1). 
LUCAS G. 1997 - Les lamelles Dufour du Flageolet I (Bézenac, Dordogne) dans le contexte aurignacien. Paléo, 9, p. 191-219.

LUCAS G. 1999 - Production expérimentale de lamelles torses : approche préliminaire, Bulletin de la Société Préhistorique Française, 96 (2), p. 145-151.

LUCAS G. 2000 - Les industries lithiques du Flageolet (Dordogne) : approche économique, technologique, fonctionnelle et analyse spatiale. Thèse de Doctorat, Université de Bordeaux I, $600 \mathrm{p}$.

PELEGRIN J., O'FARRELL M. 2005 - Les lamelles retouchées et utilisées de Castanet. In : F. Le BrunRicalens, J-G. Bordes, F. Bon (ed.), Productions lamellaires attribuées à l'Aurignacien : chaînes opératoires et perspectives techno-culturelles. XIV ìme congrès de I'Union internationale des sciences préhistoriques et protohistoriques, Liège (2-8 septembre 2001). Luxembourg : MNHA, p. 103-121 (ArchéoLogiques, 1).

PELEGRIN J., WHITE R. 1998 - Abri Castanet (Sergeac, Dordogne). Rapport de fouille programmée triannuelle (1996-1998). Rapport remis au SRA Aquitaine.

PELEGRIN J., WHITE R. 1999 - Sergeac, Castelmerle. Bilan scientifique de la région Aquitaine, 1998. Bordeaux : Service régional de l'Archéologie, Drac Aquitaine, Ministère de la Culture et de la Communication, p. 37.

PEYRONY D. 1935 - Le gisement Castanet, Vallon de Castelmerle, commune de Sergeac (Dordogne), Aurignacien I et II. Bulletin de la Société Préhistorique Française, 32, p. 418-443.

RIGAUD J.-P. 1993 - L'Aurignacien dans le Sud-Ouest de la France. Bilan et perspectives. In : L. Bánesz, J.-K. Kozlowski, actes du XII Congrès International des Sciences Préhistoriques et Protohistoriques 2, Institut Archéologique de l'Académie Slovaque des Sciences, Bratislava, 1-7 septembre 1991, p. 181-186.
SONNEVILLE-BORDES D. de 1960 - Le Paléolithique supérieur en Périgord. Bordeaux : Delmas, 555 p.

TIXIER J. 1991 - Champ Parel, Corbiac-Vignoble 2 (CV2). Gallia Informations, circonscription Aquitaine, 1, Paris : éditions du CNRS, p. 8-10.

TIXIER J., INIZAN M.-L., ROCHE H. 1980 - Terminologie et technologie. Antibes: Cercle de recherches et d'études préhistoriques, 120 p. (Préhistoire de la pierre taillée, 1).

WHITE R. 2007 - Sergeac. L'abri Castanet. Bilan scientifique de la région Aquitaine, 2005. Bordeaux : Service régional de l'Archéologie, Drac Aquitaine, Ministère de la Culture et de la Communication, p. 52.

WHITE R. 2008 - Sergeac. Abri Castanet. Bilan scientifique de la région Aquitaine, 2006. Bordeaux : Service régional de l'Archéologie, Drac Aquitaine, Ministère de la Culture et de la Communication, p. 42-44.

WHITE R. 2009 - Sergeac. Abri Castanet. Bilan scientifique de la région Aquitaine, 2007. Bordeaux : Service régional de l'Archéologie, Drac Aquitaine, Ministère de la Culture et de la Communication, p. 60-61.

WHITE R., MENSAN R., SISK M., CLARK A. 2010 Sergeac. Abri Castanet. Bilan scientifique de la région Aquitaine, 2008. Bordeaux : Service régional de l'Archéologie, Drac Aquitaine, Ministère de la Culture et de la Communication, p. 52-55. 
